\title{
Applicability of the DFT Augmented Symmetry Approach to Simulations of the Chromium-based Rings: Cross-validation Using the PBE Functional
}

\author{
Michał Wojciechowski $^{*}$, Bartosz Brzostowski ${ }^{1}$, Grzegorz Kamieniarz ${ }^{2}$ \\ ${ }^{1}$ Institute of Physics, University of Zielona Góra, \\ Prof. Szafrana 4a, 65-516 Zielona Góra, Poland \\ ${ }^{2}$ Faculty of Physics, A. Mickiewicz University \\ Umultowska 85, 61-614 Poznań, Poland \\ *E-mail:20000339@stud.uz.zgora.pl
}

Received: 10 May 2016 ; accepted: 28 May 2016 ; published online: 16 June 2016

\begin{abstract}
We present comprehensive convergence and accuracy tests for predictions of the augmented symmetry approach suggested to reduce computational complexity of the DFT calculations for molecular rings. Using the PBE functional, we demonstrate the numerical stability of magnetic couplings, magnetic moments and the HOMO-LUMO gaps with respect to the size of the basic parameters RKM, the number of k-points and types of the unit cells as well as to the presence of full or absence of any point group symmetry. We show that both the performance and the final results are equal to those obtained for the standard approaches but the computing time is significantly lower. We conclude that the value RKM $=3.0$ and a single k-point in the irreducible Brillouin zone are enough to reach the uncertainty of magnetic couplings of the order of $0.1 \mathrm{meV}$ and a distortion resulting from the approach is irrelevant as far as the magnetic properties are concerned.
\end{abstract}

Key words: molecular magnets, chromium nanorings, density functional theory

\section{INTRODUCTION}

The density functional theory is an extremely important first principle approach and through the past several decades has become one of the fundamental calculation methods in physics, chemistry and many interdisciplinary studies. Knowledge of first principles allows us to search for solutions of Hamilton equations describing complex systems. Development of computer technologies allowed us to perform the first principle calculations numerically, effectively and fast. However, our demands grow along with that progress. We would like to perform calculations faster, easier, with greater precision for bigger, more complex systems. These demands can be fulfilled either on hardware level, by creating faster computers or on software level, by creating better mathematical models and algorithms. There is also another approach - using internal symmetry within a studied compound to optimize input data.
Generally one can perform the first principle calculations without using symmetry, which means that each atom in the structure has unique representation. This approach can be applied to any system (crystal, slab, molecule); however, it may be problematic for large systems due to computational requirements. In opposition to that, instead of encoding the whole structure from unique atoms, one uses smaller cells to reconstruct the molecule using symmetries. It can successfully reduce the size of the structure, but it has one considerable flaw. This approach can be applied to any structure as long as it is invariant under some symmetry operations. As a result, systems without symmetry cannot be simplified. However, for those systems one can perform some artificial modifications in order to force the symmetry onto the structure by replacing the actual system with an approximate one that mimics its topology and overall geometry $[1,2]$. 
In this paper we investigate the $\mathrm{Cr}_{7} \mathrm{M}$ family of molecular rings using the WIEN2k package in order to conduct the cross-validation of our augmented symmetry approach as well as to check convergence, numerical stability of the predictions and their accuracy with respect to well-established data in literature. This is needed for future improvement of the approach in conjunction with the hybrid functionals or for envisaged applications in designing deposition of magnetic molecules on a surface [3] or their realizations as molecular junctions [4].
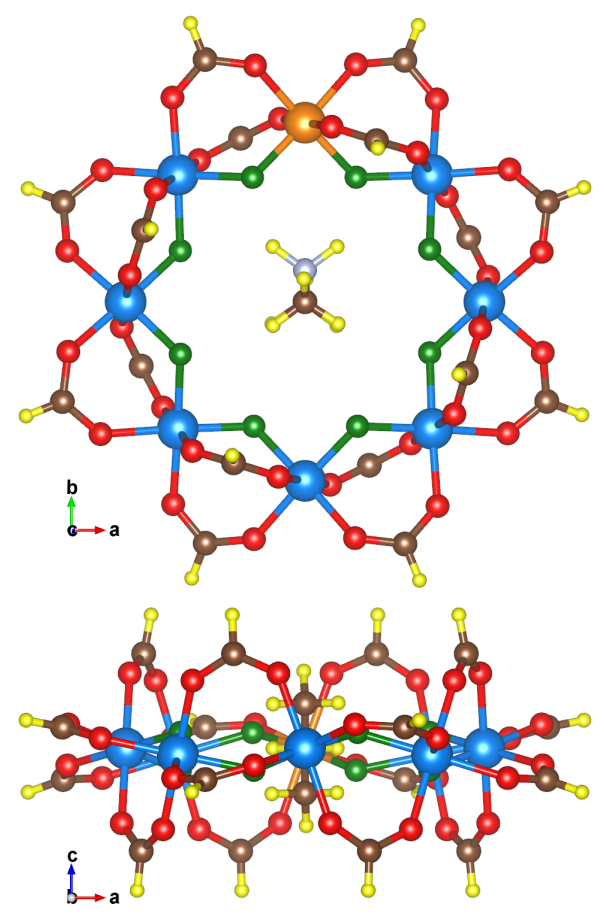

Fig. 1. Simplified structure of the $\mathrm{Cr}_{7} \mathrm{M}$ molecule. Hydrogen atoms are shown in place of pivalic groups. Chromium is blue, oxygen is red, carbon is brown, fluorine is green, hydrogen is yellow and nitrogen is gray. Orange represents the substituting atom $\mathrm{M}=\mathrm{Cr}, \mathrm{Cd}, \mathrm{Ni}$

\section{STUDIED MOLECULES}

We carry out the tests for three molecular nanowheels: $\mathrm{Cr}_{8} \mathrm{~F}_{8}(\mathrm{Piv})_{16}, \mathrm{Cr}_{7} \mathrm{CdF}_{8}(\mathrm{Piv})_{16}$ and $\mathrm{Cr}_{7} \mathrm{NiF}_{8}(\mathrm{Piv})_{16}[5,6]$. For short we refer to them as $\mathrm{Cr}_{8}, \mathrm{Cr}_{7} \mathrm{Cd}$ and $\mathrm{Cr}_{7} \mathrm{Ni}$ (see Fig. 1). The Piv group is the pivalic acid - trimethyl acetic acid $\mathrm{CO}_{2} \mathrm{C}\left(\mathrm{CH}_{3}\right)_{3}$. The homonuclear $\mathrm{Cr}_{8}$ ring is built up of eight $\mathrm{Cr}^{\mathrm{III}}$ ions. They lay almost in a single layer ( $x y$ plane) slightly alternating along the $z$ axis and their positions determine the highest symmetry among the $\mathrm{Cr}_{7} \mathrm{M}$ molecules. Neighboring pairs of chromium atoms are connected by a single fluorine bridge oriented inside a ring and two pivalic groups that span outside.

Heteronuclear derivatives are created by substitution of one chromium atom for cadmium or nickel which destroys the symmetry. Furthermore, there is a small nonmagnetic molecule $\mathrm{NH}_{2}\left(\mathrm{CH}_{3}\right)_{2}$ situated inside the ring which can be neglected for the homonuclear ring but is needed for the heteronuclear derivatives to preserve the charge balance. When the $\mathrm{Cr}^{\mathrm{III}}$ ion is substituted by a $\mathrm{Cd}^{\mathrm{II}}$ or $\mathrm{Ni}^{\mathrm{II}}$ ion, the ring loses one electron. The bonds of substituting ion become unsaturated and the whole molecule loses approximately $1 \mu_{B}$ of magnetic momentum. The inner molecule donates a single electron to the ring saturating the bond. $\mathrm{The} \mathrm{Cr}_{7} \mathrm{M}$ molecule (where $\mathrm{M}$ is $\mathrm{Cr}, \mathrm{Cd}$ or $\mathrm{Ni}$ ) is presented in Fig. 1 from two perspectives.

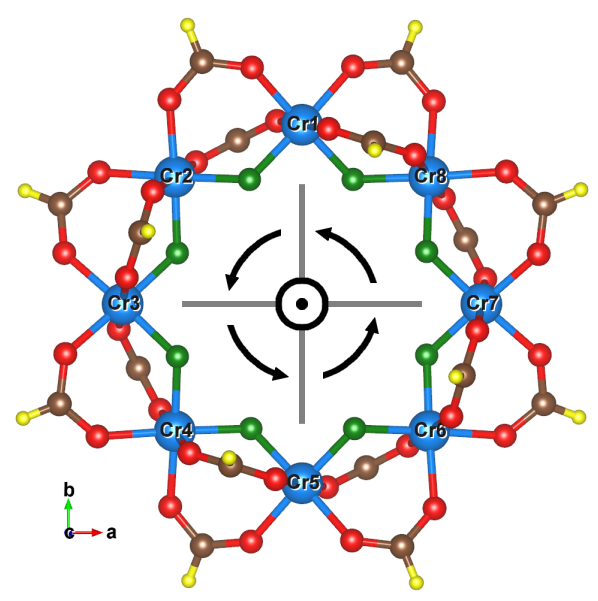

Fig. 2. Schematic representation of the rotational symmetry in $\mathrm{Cr}_{8}$ molecule. $\mathrm{Cr} 1$ and $\mathrm{Cr} 2$ (along with accompanying bridges) constitute unique inequivalent atoms. Atoms $\mathrm{Cr} 3, \mathrm{Cr} 5$ and $\mathrm{Cr} 7$ are equivalent

to $\mathrm{Cr} 1$ and atoms $\mathrm{Cr} 4, \mathrm{Cr} 6$ and $\mathrm{Cr} 8$ are equivalent to $\mathrm{Cr} 2$

In order to decrease the number of atoms considered in the ring from 272 to 80 , while keeping the magnetic properties unaffected, a process referred to as hydrogen saturation is employed $[2,8,9]$. Each methyl group $\mathrm{CH}_{3}$ of the pivalic group is substituted by a single $\mathrm{H}$ atom. This replaces $\mathrm{CO}_{2} \mathrm{C}\left(\mathrm{CH}_{3}\right)_{3}$ with $\mathrm{CO}_{2} \mathrm{CH}_{3}$. By applying this process once more we obtain $\mathrm{O}_{2} \mathrm{CH}$ bridge which replaces the whole pivalic group.

The crystallographic structures of the studied compounds have been deposited in Cambridge Crystallographic Data Centre (CCDC) [7]. Records CCDC-164814 and CCDC-164814 through CCDC-191623 contain the supplementary crystallographic data used for this paper. These data can be obtained free of charge at CCDC website: www.ccdc.cam.ac.uk/conts/retrieving.html.

\section{AUGMENTED SYMMETRY APPROACH}

The $\mathrm{Cr}_{8}$ molecule possess full rotational symmetry (FS) and can be reconstructed by rotating a quarter of the ring containing two metallic ions around the axis perpendicular to the plane of the molecule. This symmetry is presented in 
Fig. 2. Within the WIEN2k package symmetry puts artificial constrains on the system in question by reducing the number of inequivalent atoms. In turn, the number of degrees of freedom declines which means that fewer steps have to be made before self-consistency is reached. Also an execution of a single iteration takes less time. All of these lead to significantly faster accomplishment of convergence of calculations. Unfortunately, for heteronuclear derivatives substitution of a single chromium ion with another element removes the symmetry completely and the number of degrees of freedom cannot be reduced.

This problem of an excessive number of inequivalent atoms and a very slow convergence of DFT calculations for heterometallic rings $\mathrm{Cr}_{7} \mathrm{M}$ had led us to propose the augmented symmetry approach (AS) [1]. In this approach the basic symmetry element for $\mathrm{Cr}_{7} \mathrm{M}$ originates from a half of the ring containing five metallic ions forming an arc. This symmetry is presented in Fig. 3. This allows us to place the doping ion $\mathrm{M}$ on one of the ends of that arc. The whole molecule is then reconstructed by rotating that arc around an axis going through its ends. While a ring with AS has more degrees of freedom than the ring with FS, their number is smaller than that of the ring with no symmetry (NS) which substantially speeds up calculations.

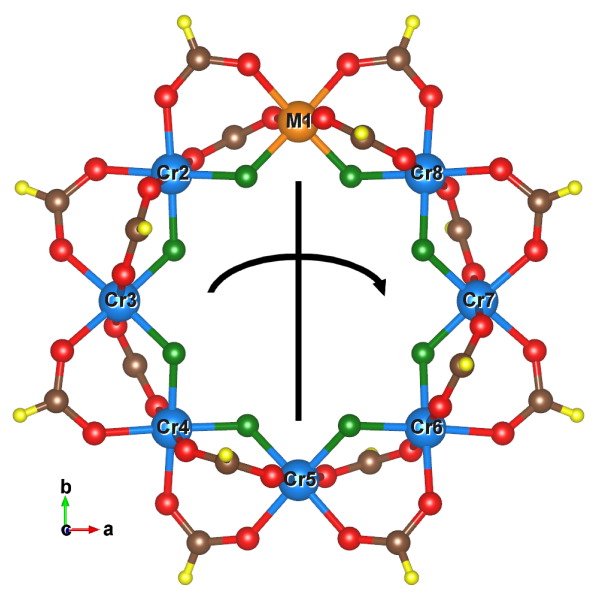

Fig. 3. Schematic representation of the augmented symmetry operation in $\mathrm{Cr}_{7} \mathrm{M}$ molecule. $\mathrm{Cr} 1, \mathrm{Cr} 2, \mathrm{Cr} 3, \mathrm{Cr} 4$ and $\mathrm{Cr} 5$ (along with accompanying bridges) constitute an arc composed of unique, inequivalent atoms. The atoms $\mathrm{Cr} 2, \mathrm{Cr} 3$ and $\mathrm{Cr} 4$ are equivalent to $\mathrm{Cr} 8$, $\mathrm{Cr} 7$ and $\mathrm{Cr} 6$, respectively

Unfortunately, the AS approach effectuates small distortion into the ring (see [1] for more details). The resulting AS structure does not exactly match the original $\mathrm{Cr}_{7} \mathrm{M}$ molecule. In the AS model some chromium ions are slightly shifted from their real positions (see Fig. 3 in [1]). This mismatch is the reason why the predictions of the AS model need to be carefully checked, as far as the convergence and the numer- ical accuracy of the electronic and magnetic properties are concerned.

\section{COMPUTATIONAL DETAILS}

The $a b$ initio calculations based on the FS, AS and NS model are carried out using density functional theory (DFT) $[10,11]$ as implemented in WIEN2k computational package [12-14]. It uses the all electron linearized augmented plane wave (LAPW) method $[15,16]$. The core and valence states are treated separately. The core states are treated fully relativistically [17]. Relativistic effects are also included for valence electrons by scalar relativistic treatment [18]. In our calculations we use a default value of -6.0 Ry to separate them. The chromium core states are those from neon configuration (1s2s2p). This leaves us with the states $3 \mathrm{~s}, 3 \mathrm{p}, 3 \mathrm{~d}$ and $4 \mathrm{~s}$ which are considered valence. For cadmium the core configuration is defined as $1 \mathrm{~s} 2 \mathrm{~s} 2 \mathrm{p} 3 \mathrm{~s} 3 \mathrm{p} 3 \mathrm{~d} 4 \mathrm{~s}$ whereas $4 \mathrm{p} 4 \mathrm{~d} 5 \mathrm{~s}$ is the valence configuration. For nickel the core configuration is defined as $1 \mathrm{~s} 2 \mathrm{~s} 2 \mathrm{p} 3 \mathrm{~s}$ whereas $3 \mathrm{p}, 3 \mathrm{~d}$ and $4 \mathrm{~s}$ states are considered valence ones.

The LAPW method incorporates muffn-tin radii $\left(r_{m t}\right)$ approximation [19]. According to this approximation around each atom in the structure there is a spherical area called atomic sphere. All electrons within atomic spheres are considered to belong to atoms encompassed by those spheres. Atomic spheres cannot overlap or be too small, so that the core electrons do not leak out. This is very important, because within atomic spheres electrons are described with wave functions that can be expressed as a product of spherically symmetrical radial function $R(r)$ and spherical harmonics $Y_{l m}$. The area outside atomic spheres is called interstitial region and all electrons contained in it are described by the plain wave functions. For consistency we use here the same values of the $r_{m t}$ parameters as in our previous study [1,20] which in turn are identical to those chosen elsewhere [9]. They are presented in Tab. 1.

Tab. 1. Values of muffin-tin radii $r_{m t}$ for different elements. All values in units of $r_{\text {Bohr }}$

\begin{tabular}{l||r|r|r|r|r|r|r}
\hline element & $\mathrm{Cr}$ & $\mathrm{Cd}$ & $\mathrm{Ni}$ & $\mathrm{F}$ & $\mathrm{O}$ & $\mathrm{C}$ & $\mathrm{H}$ \\
\hline \hline$r_{m t}$ & 2.40 & 2.40 & 2.40 & 1.20 & 1.24 & 1.00 & 0.83 \\
\hline
\end{tabular}

If not stated otherwise, the parameter defining the basis set is RKM $=3.0$, which allows for density matrix size (number of augmented plane waves) of the order of 35000 . We account for the exchange and correlation effects using generalized gradient approximation implemented in the Perdew, Burke and Ernzerhof (PBE) functional [21]. We do not include spin-orbit coupling in our calculations, as it was already shown that the differences in the results are negligible $[2,9]$ and the single-ion anisotropy for the $\mathrm{Cr}$ ions is known to be very small $[6,22,23]$. 


\section{1. Magnetic moments and HOMO-LUMO gaps}

Two electronic densities are considered in our spinpolarized calculations: one for spin channel up and one for spin channel down. Through integration of these densities (expressed in units of $e / r_{\text {Bohr }}^{3}$ ) for specific atoms within appropriate atomic spheres one can calculate electric charges (expressed in units of $e$ ). By integrating the up and down densities, we obtain $Q_{\text {up }}$ and $Q_{\text {down }}$ charges. Total electric charges $Q_{\text {tot }}$ are obtained by summing the results, i.e. $Q_{\text {tot }}=Q_{\text {up }}+Q_{\text {down }}$. In a similar fashion magnetic moments $M_{m}$ (expressed in units of $\mu_{B}$ ) can be calculated as a difference of charges $M_{m}=Q_{\text {up }}-Q_{\text {down }}$.

The HOMO-LUMO (H-L) gap is defined as energy difference between frontier orbitals: the highest occupied molecular orbital (HOMO) and the lowest unoccupied molecular orbital (LUMO). This gap can be used to predict the strength and stability of transition metal complexes. The H-L gap within organic compounds is an equivalent of a band gap in inorganic semiconductors. Within the standard method H-L gaps are easy to extract from DFT results. One needs only KohnSham levels (KSL) and Fermi level (FL). The HOMO is the first orbital under FL and LUMO is the first orbital above FL. By subtracting energies of these orbitals one obtains the H-L gap. When conducting the study of magnetic properties, there are two separate sets of KSL, one for spin up channel and one for spin down channel. The spin up electrons are the majority charge carriers and spin down electrons are the minority charge carriers. The H-L gaps for these channels usually differ and as a result we obtain two separate types of the H-L gaps.

We note that theoretical interpretation of the gaps is still problematic [4, 24, 25]. The PBE-based DFT results often underestimate the expected values. They can be corrected using better correlation and exchange functional or by performing two sets of calculations: one for the neutral $N$ particle system and one for the charged $N+1$ particle system [24, 26, 27]. Within the scope of this paper we restrict ourselves to the standard method, subtracting the corresponding energies.

\section{2. Exchange couplings}

The magnetic coupling parameters $J$ between transition metals in a ring are estimated within the standard projected broken symmetry approach (PBS) [28], considering the Isinglike magnetic Hamiltonian

$$
H_{\mathrm{mag}}=\sum_{i}^{N} J_{i} \sigma_{i} \sigma_{i+1},
$$

where $N=8$ is the number of magnetic ions in the ring, $\sigma_{i}$ is the classical spin variable at site $i$ assuming the values $\sigma_{i}= \pm S_{i}$, which is subject to the periodic boundary condition, i.e. $\sigma_{i}=\sigma_{i+N}$ and $J_{i}$ is the nearest-neighbor coupling between two ions at positions $i$ and $i+1$.
For chromium ions the spin variable is equal to $S=\frac{3}{2}$, for cadmium $S=0$ and nickel has $S=1$. We consider that for a given ring, all the couplings between chromium ions $J_{i}$ are the same so that $J_{i}=J$. The coupling between chromium and nickel is denoted $J^{\prime}$ whereas that between chromium and non-magnetic cadmium is missing. The interaction schemata are shown in Fig. 4.

Within the AS model the $\mathrm{Cr}_{7} \mathrm{M}$ rings have five nonequivalent transition metal ions. This allows for 9,16 and 8 nonequivalent spin configurations for $\mathrm{Cr}_{8}, \mathrm{Cr}_{7} \mathrm{Ni}$ and $\mathrm{Cr}_{7} \mathrm{Cd}$, respectively. By fitting the energy level structure arising from Equation 1 to the DFT energies obtained for all nonequivalent spin configurations (performing multivariate linear regression like in [29]), we extract the coupling constants. Within the $\mathrm{NS}$ model the $\mathrm{Cr}_{7} \mathrm{M}$ rings have eight nonequivalent transition metal ions. This allows for 18,72 and 36 nonequivalent spin configurations for $\mathrm{Cr}_{8}, \mathrm{Cr}_{7} \mathrm{Ni}$ and $\mathrm{Cr}_{7} \mathrm{Cd}$, respectively. For this slowly convergent approach the coupling constants were extracted using frontier spin configurations: antiferromagnetic (AF) and ferromagnetic (FM). The formulae exploited are explicitly given in Section 4 of our preceding publication [1]. For $\mathrm{Cr}_{7} \mathrm{Ni}$, apart from the standard frontier configurations, an extra configuration is needed. It is referred to as AM and corresponds to the nickel spin reversed with respect to that in AF. For the FS model the $\mathrm{Cr}_{8}$ ring has two nonequivalent transition metal ions and 2 nonequivalent spin configurations: AF and FM. This leads to the regression equation identical to a formula used in [1].

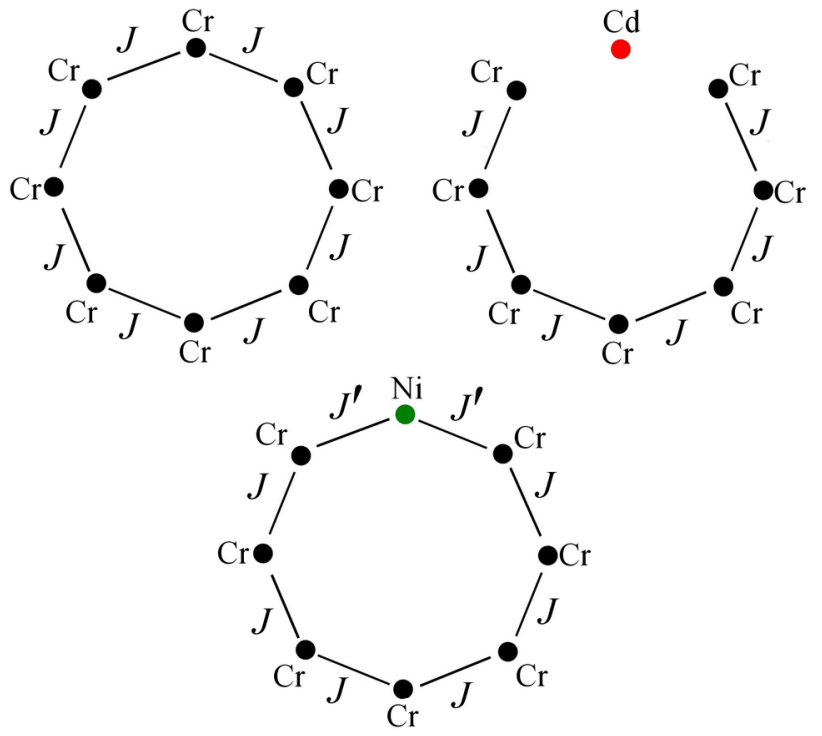

Fig. 4. Schematic representations of exchange couplings between the ions for $\mathrm{Cr}_{8}$ (top left), $\mathrm{Cr}_{7} \mathrm{Cd}$ (top right) and $\mathrm{Cr}_{7} \mathrm{Ni}$ (bottom) molecule. Black dots represent $\mathrm{Cr}^{\mathrm{III}}$ ions, the red dot represents $\mathrm{Cd}^{\mathrm{II}}$ ion and the green dot represents $\mathrm{Ni}^{\mathrm{II}}$ ion

In our study we take into account only interactions between the nearest neighboring ions. The exchange interaction 
between transition metal ions in a ring is not direct. The actual exchange is carried through superexchange paths laid by the carboxylate and fluorine bridges. As a result any interaction between ions not connected by those bridges is virtually impossible.

\section{NUMERICAL TESTS AND CONVERGENCE CRITERIA}

For our study there are two types of convergence tests considered. The first one is the convergence of results in a single run which we designate as internal convergence. Within the DFT study one considers the spin density of electrical charge. The calculations start with specific numerical parameters and from some initial theoretical density dependent on elements which constitute the system. In a single iteration (single self-consistent cycle) the following operations are performed. First the physical properties for current electronic density are calculated with the help of a given correlation and exchange functional (in our case PBE). Then the Hessian matrix which is used to modify the existing density is obtained. Finally, the convergence criteria are checked. If they are met the calculations are finished, otherwise the next iteration begins. As these criteria we choose the energy change and the charge distance defined in WIEN2k. Energy change is simply an average value of change in total energy of a system over the last three iterations. Charge distance is the average change of electrical charge within the muffin tins of the $L=0 \mathrm{com}$ ponent of the original and modified density. It tends to zero at the fixed-point solution which is the variational minimum of the energy. The energy change and the largest value of charge distance for all the atoms in a structure are compared to the threshold values. These values are 0.0001 Ry for energy and $0.0001 \mathrm{e}$ for charge and they constitute very good internal convergence criteria.

The second type of convergence (which we designate as external convergence) is the convergence with respect to the parameters used in different computational runs. When performing calculations, numerical parameters used in the models and their implementations act as a sort of artificial variable $v_{a}$. In order to view the obtained data as objective results denoted by $r$, one has to be sure that these results do not depend on those variables. As this criterion can never be truly met, it is reformulated in a different manner: the obtained results are considered numerically valid if for some range of parameters they are stable. This stability is understood as an insignificant change of results with respect to used parameters, which leads to

$$
\frac{\partial r\left(v_{a}\right)}{\partial v_{a}} \approx 0
$$

For the purpose of this paper we mainly concentrate on tests of the external convergence for the exchange coupling $J$ with respect to RKM parameter, k-mesh and cell size. The tests are performed for $\mathrm{Cr}_{8}$ molecule using the FS, NS and AS approach. The NS data are considered as the reference point.

\section{1. Convergence with respect to RKM and k-mesh}

The most important parameter in the LAPW method is RKM (or RKMAX) [30]. RKM stands for the product of the smallest atomic sphere radius $r_{m t}$ times the largest $\mathrm{K}$-vector $k_{\text {max }}$ (of the plane wave expansion of the wave function). It thus determines the size of the basis set (number of plane waves), size of the matrices, the accuracy and consequently the amount of necessary computer time. Larger RKM values lead to more accurate calculations; however, the computing time scales with RKM as RKM ${ }^{3}$. Proper RKM values depend on the desired accuracy for a given property, the size of the atomic spheres and the specific atom with the smallest $r_{m t}$. The values between 6-10 are usually reasonable but for systems with large differences between $r_{m t}$ sizes they may lead to unphysical eigenvalues. Systems containing hydrogen with short bond length and thus a very small $r_{m t}$ are a special case and usually require a much smaller RKM value around 3. Nonetheless convergence must be checked for a new type of system or approach [31].

Similarly the size of k-mesh is important [30]. It determines the number of points used for numerical integration. In general, metallic systems need a fine mesh while for insulators very few k-points are often sufficient. Small unit cells need a fine mesh, while large unit cells most likely do not. Supercells for surfaces (slabs) need only one k-point along the direction perpendicular to the surface. Insulators with many atoms per cell may need only one shifted k-point. Supercells for single atoms or molecules should be done just with a single central point. While there are general recommendations on how to choose the k-mesh, the specific values are difficult to predict. The necessary number of k-points must always be verified by first doing a calculation with a crude mesh and then continue with a fine mesh and comparing the results [31].

Variations of the values of $J$ parameter with respect to RKM and the number of k-points are shown in Tab. 2 for the NS and FS approach, and in Tab. 3 for the AS approach. When analyzing the $J$ values for a given RKM and different number of k-points listed in Tab. 2 and Tab. 3, we can see that for $\mathrm{RKM}=2.25$ and upwards the $J$ values vary within $0.1 \mathrm{meV}$ range, which is within the numerical uncertainty of the expected and satisfying results. Also it is clear that the number of k-points does not influence the accuracy of the couplings so that a single central k-point is sufficient. As for the RKM dependence of $J$ we see that for $\mathrm{RKM}=2.5$ and upwards the variation is also negligible. It is also clear that the AS model assumptions have no influence on the stability and accuracy of the results for $J$. We infer that RKM $=3.0$ is the proper choice for reliable applications of our DFT method. 
Tab. 2. Exchange interaction parameter $J$ obtained for $\mathrm{Cr}_{8}$ molecule in the NS approach for different RKM values and number of kpoints. In the last column the data calculated in the FS approach for different RKM values and one k-point are inserted. All the values in units of $\mathrm{meV}$

\begin{tabular}{l||r|r|r|r|r}
\hline RKM & $1 k_{p}$ & $8 k_{p}$ & $10 k_{p}$ & $30 k_{p}$ & FS, $1 k_{p}$ \\
\hline \hline 2.00 & 7.33 & 7.08 & 7.19 & 7.08 & 7.40 \\
\hline 2.25 & 7.08 & 7.02 & 6.99 & 7.00 & 7.06 \\
\hline 2.50 & 6.78 & 6.73 & 6.73 & & 6.80 \\
\hline 2.75 & 6.59 & 6.57 & & & 6.62 \\
\hline 3.00 & 6.75 & & & & 6.75 \\
\hline
\end{tabular}

Tab. 3. Exchange interaction parameter $J$ obtained for $\mathrm{Cr}_{8}$ molecule in the AS approach for different RKM values and number of k-points. All the values in units of $\mathrm{meV}$

\begin{tabular}{l||c|c|c|c}
\hline RKM & $1 k_{p}$ & $8 k_{p}$ & $10 k_{p}$ & $30 k_{p}$ \\
\hline \hline 2.00 & 7.40 & 7.20 & 7.26 & 7.22 \\
\hline 2.25 & 7.05 & 6.98 & 7.02 & 7.01 \\
\hline 2.50 & 6.77 & 6.72 & 6.74 & \\
\hline 2.75 & 6.56 & 6.56 & & \\
\hline 3.00 & 6.71 & & & \\
\hline
\end{tabular}
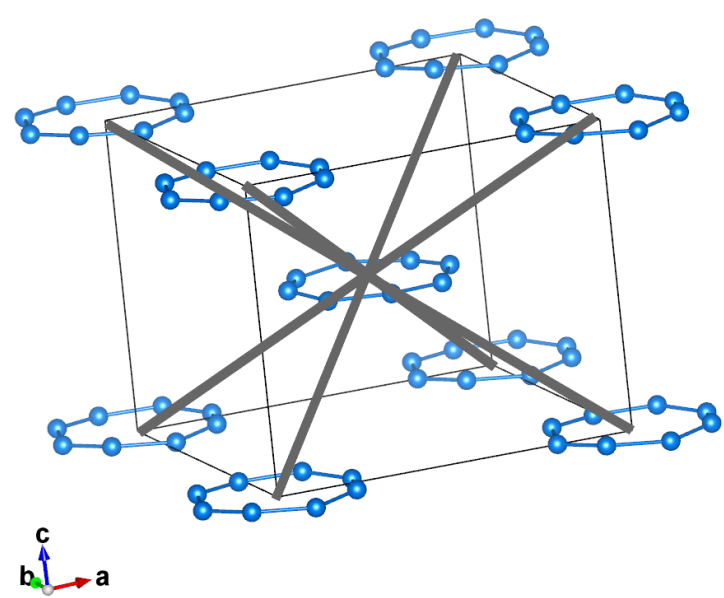

Fig. 5. Placement and orientation of the nearest neighbor molecules in the original unit cell. Each molecule is coordinated with eight other molecules placed in direction of the corners of the base cell

\section{2. Convergence with respect to cell size}

An original unit cell, as measured in experiment [5], contains two $\mathrm{Cr}_{8}$ ring molecules. Parameters of this unit cell are $a=20.09289 \AA, b=20.09289 \AA, c=16.80091 \AA$, $\alpha=90^{\circ}, \beta=90^{\circ}, \gamma=90^{\circ}$. The volume of the entire cell is $V=6782.93 \AA^{3}$ and the volume per one ring is $V_{m}=3391.47 \AA^{3}$. The two molecules are placed on two separate layers separated by a distance of $8.400455 \AA$, which is simply half of $c$. The distance between centers of those molecules is around $16.26 \AA$ and the smallest distance between magnetic ions of two rings is $9.54 \AA$. In our study we concentrate on a single molecule therefore we modify the structure of the cell. We remove one molecule, changing orientation of the nearest molecules in relation to each other as shown in Figures 5 and 6. We also change the size of the cell. We keep the angles unchanged $\left(\alpha=\beta=\gamma=90^{\circ}\right)$ and relation between $a$ and $b(a=b)$.

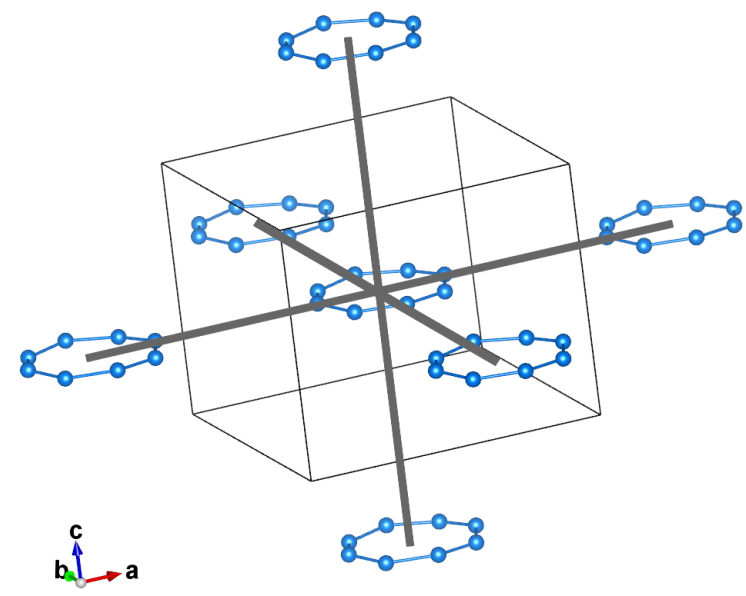

Fig. 6. Placement and orientation of the nearest neighbor molecules in the modified base cell. Each molecule is coordinated with six other molecules placed in direction of the walls of the base cell

In our analysis we introduce five cell sizes based on different assumptions:

- cell A: $a=20.09 \AA, c=16.80 \AA, V=6781 \AA^{3}$. This cell has the same size as the original one. Since the orientation of the molecules is different, keeping the same size means that the distance between centers of nearest molecules is the same;

- cell B: $a=20.09 \AA, c=13.80 \AA, V=5248 \AA^{3}$. This smaller cell is meant to slightly decrease (by $3 \AA$ A) the distance along $c$;

- cell C: $a=20.09 \AA, c=9.54 \AA, V=3852 \AA^{3}$. The distance between rings on a single layer is unchanged but the distance between two layers is now the same as the smallest distance between magnetic ions of two rings in the original cell;

- cell D: $a=18.62 \AA, c=9.78 \AA, V=3391 \AA^{3}$. The distance between centers of the molecules on a single layer and between layers is now the same $(9.78 \AA)$. Also the total cell volume (which is also the volume per molecule) is now the same as volume per molecule in the original cell;

- cell E: $a=20.09 \AA, c=8.40 \AA, V=3391 \AA^{3}$. This cell was obtained by simply cutting the $c$ length of the original cell by half. The volume per molecule remains the same. Distances between molecules on a single layer and distances between layers are also unchanged. However, the distance between molecules on neighbor- 
ing layers is now clearly smaller than in the original molecule.

For all the cells considered we have performed tests, verifying how the estimates of $J$ parameter change with RKM. The results are presented in Tab. 4. It is clear that the cell size does not really influence the values of $J$ even for cell $\mathbf{E}$ which has the tighter stacking of the rings on top of each other. Results are identical as long as there is a sufficient distance between molecules to treat them as separate entities and not as parts of a larger structure.

Tab. 4. Exchange interaction parameter $J$ for different unit cells and RKM values found within the AS approach. All the values in units of meV

\begin{tabular}{l||r|r|r|r|r}
\hline RKM & A & B & D & C & E \\
\hline \hline 2.00 & 7.18 & 6.99 & 7.10 & 6.94 & 6.90 \\
\hline 2.25 & 7.05 & 6.72 & 6.81 & 6.78 & 6.71 \\
\hline 2.50 & 6.69 & 6.66 & 6.64 & 6.61 & 6.69 \\
\hline 2.75 & 6.56 & 6.59 & 6.65 & 6.50 & 6.67 \\
\hline 3.00 & 6.71 & 6.77 & 6.73 & 6.77 & 6.75 \\
\hline
\end{tabular}

\section{3. Computational complexity}

Calculations were carried out on a supercomputing platform consisting of identical machine nodes with Intel Xeon E5-2697 v3 @ 2.60GHz processors (haswell group of Inula cluster at PSNC). We have measured execution time and the number of iterations needed to reach the convergence for different symmetries, spin configurations and values of RKM. We have also performed these tests with different numbers of assigned cores in order to analyze the scalability of all approaches from this point of view and to demonstrate the advantages of the AS idea. The AS temporal complexity is remarkably diminished with respect to the NS requirements and is only slightly higher than that for the FS treatment. The out- comes of our profound computational complexity analysis are presented in Tables 5 and 6 .

As it was expected, the average execution time of a single iteration $t_{\text {iter }}$ was the longest for the NS approach and the shortest for FS. Performance of the AS approach is settled on between these two, but closer to FS. For RKM $=3.0$ all the values of $t_{\text {iter }}$ become closer together as we increase the number of cores, but the general hierarchy is still kept. However, the very value of $t_{\text {iter }}$ is not so important. The total time $T_{\text {tot }}$ of calculations really matters, as the average number of iterations $N$ necessary to reach convergence is much greater for the NS approach than that of AS or FS approach. For NS, as shown before, the number of iterations increases significantly with enhancing RKM. While for RKM $=2.0$ we had no problem in running the NS calculations, for $\mathrm{RKM}=2.5$ we failed. Only for one spin configuration we managed to reach convergence in 128 steps. The computational runs for remaining spin configurations were stopped as the number of iterations reached the allowed limit equal to 150 . We have not attempted to run calculations for RKM $=3.0$. It became clear that the convergence for the NS could not be reached within a reasonable time period. Concluding, the AS approach improves the computational performance with respect to the NS calculations without any loss of stability and numerical accuracy of the final results.

\section{4. Additional testing}

For all the tests performed only the estimates of $J$ parameter were demonstrated as yet. Other quantities were also subject to verification, but there is no need to present actual results because their values coincide. The $J$ parameters were the only quantities showing any numerical variation, and even then that dependence on RKM and the number of k-points was very weak. Magnetic moments varied in a range of $\pm 0.0002 \mu_{B}$ when usually the acceptable numerical uncertainty of these results is $\pm 0.01 \mu_{B}$. HOMO-LUMO gaps

Tab. 5. Execution time of a single iteration $t_{\text {iter }}$ for different approaches applied to $\mathrm{Cr}_{8}$ molecule with respect to the number of cores. Results are given in absolute units (hours and minutes) as well as in relation to the FS approach $\left(t_{\mathrm{rel}}\right)$. The values quoted are averaged over series of iterations carried out for different spin configurations

\begin{tabular}{l|l||r|r|r|r|r|r|r|r}
\cline { 3 - 9 } \multicolumn{2}{c}{} & \multicolumn{2}{c|}{1 core } & \multicolumn{2}{c|}{2 cores } & \multicolumn{2}{c|}{4 cores } & \multicolumn{2}{c}{8 cores } \\
\hline RKM & sym. & $t_{\text {iter }}$ & $t_{\text {rel }}$ & $t_{\text {iter }}$ & $t_{\text {rel }}$ & $t_{\text {iter }}$ & $t_{\text {rel }}$ & $t_{\text {iter }}$ & $t_{\text {rel }}$ \\
\hline \multirow{3}{*}{2.0} & NS & $01: 58$ & 3.0 & $01: 37$ & 3.6 & $01: 28$ & 4.4 & $01: 26$ & 5.1 \\
& AS & $01: 02$ & 1.6 & $00: 45$ & 1.7 & $00: 42$ & 2.1 & $00: 46$ & 2.7 \\
& FS & $00: 40$ & 1.0 & $00: 27$ & 1.0 & $00: 20$ & 1.0 & $00: 17$ & 1.0 \\
\hline \multirow{3}{*}{2.5} & NS & $05: 53$ & 1.6 & $03: 44$ & 2.6 & $02: 49$ & 2.3 & $02: 30$ & 2.8 \\
& AS & $04: 15$ & 1.2 & $02: 53$ & 2.0 & $01: 39$ & 1.3 & $01: 49$ & 2.0 \\
& FS & $03: 39$ & 1.0 & $01: 25$ & 1.0 & $01: 14$ & 1.0 & $00: 54$ & 1.0 \\
\hline \multirow{3}{*}{3.0} & NS & $18: 14$ & 1.2 & $11: 07$ & 1.4 & $08: 40$ & 1.5 & $05: 15$ & 1.2 \\
& AS & $16: 37$ & 1.1 & $09: 31$ & 1.2 & $06: 49$ & 1.2 & $04: 20$ & 1.0 \\
& FS & $14: 50$ & 1.0 & $08: 07$ & 1.0 & $05: 55$ & 1.0 & $04: 30$ & 1.0 \\
\hline
\end{tabular}


Tab. 6. Number of iterations $N$ necessary to reach convergence and total execution time $T_{\text {tot }}$ for all the approaches applied to $\mathrm{Cr}_{8}$ molecule with respect to number of cores. Results are given in absolute units (hours) as well as in relation to the FS approach ( $\left.T_{\text {rel }}\right)$. The values quoted are averaged over a number of different spin configurations, with the exception of those for NS approach and RKM=2.5, which were found for one configuration only

\begin{tabular}{|c|c|c|c|c|c|c|c|c|c|c|}
\hline \multirow{2}{*}{ RKM } & \multirow[b]{2}{*}{ sym. } & & \multicolumn{2}{|c|}{1 core } & \multicolumn{2}{|c|}{2 cores } & \multicolumn{2}{|c|}{4 cores } & \multicolumn{2}{|c|}{8 cores } \\
\hline & & $N$ & $T_{\text {tot }}$ & $T_{\mathrm{rel}}$ & $T_{\text {tot }}$ & $T_{\text {rel }}$ & $T_{\text {tot }}$ & $T_{\text {rel }}$ & $T_{\text {tot }}$ & $T_{\mathrm{rel}}$ \\
\hline \multirow{3}{*}{2.0} & NS & 45 & 89 & 6.6 & 73 & 8.1 & 66 & 9.9 & 65 & 11.4 \\
\hline & AS & 22 & 23 & 1.7 & 17 & 1.8 & 15 & 2.3 & 17 & 3.0 \\
\hline & FS & 20 & 13 & 1.0 & 9 & 1.0 & 7 & 1.0 & 6 & 1.0 \\
\hline \multirow{3}{*}{2.5} & NS & 128 & 753 & 9.8 & 478 & 16.1 & 361 & 13.9 & 320 & 16.9 \\
\hline & AS & 23 & 98 & 1.3 & 66 & 2.2 & 38 & 1.5 & 42 & 2.2 \\
\hline & FS & 21 & 77 & 1.0 & 30 & 1.0 & 26 & 1.0 & 19 & 1.0 \\
\hline \multirow{3}{*}{3.0} & NS & \multicolumn{9}{|c|}{ not attempted } \\
\hline & AS & 29 & 482 & 1.4 & 276 & 1.5 & 198 & 1.5 & 126 & 1.2 \\
\hline & FS & 23 & 341 & 1.0 & 187 & 1.0 & 136 & 1.0 & 104 & 1.0 \\
\hline
\end{tabular}

varied in a range of $\pm 0.1 \mathrm{eV}$ depending on RKM. These values are also within the uncertainty limit usually encountered, as visible in Tab. 7.

Tab. 7. HOMO-LUMO majority and minority gaps for the $\mathrm{Cr}_{8}$ molecule calculated for the AF and FM configurations for different symmetries, assuming $\mathrm{RKM}=3.0$

\begin{tabular}{cc||c|c|c}
\hline \multicolumn{2}{c||}{ H-L gap } & NS & AF & FS \\
\hline \hline \multirow{2}{*}{ AF } & $E_{\text {min. }}$ & 2.39 & 2.38 & 2.39 \\
& $E_{\text {maj. }}$. & 2.45 & 2.42 & 2.45 \\
\hline \multirow{2}{*}{ FM } & $E_{\text {min. }}$ & 2.22 & 2.18 & 2.22 \\
& $E_{\text {maj. }}$ & 3.80 & 3.77 & 3.80 \\
\hline
\end{tabular}

\section{RESULTS AND DISCUSSION}

In the present Section our AS results obtained for the $\mathrm{Cr}_{7} \mathrm{M}$ molecules are summarized. They are calculated using the PBE functional so that some deviations from the experimental findings can be encountered.

\section{1. Magnetic moments and HOMO-LUMO gaps}

Experimental values of magnetic moments are $2.94 \mu_{B}$ for chromium, 0 for cadmium and $2.23 \mu_{B}$ for nickel [32]. The AS results for chromium (presented in Tab. 8), while somewhat smaller, are acceptable. For cadmium the values obtained of $0.01 \mu_{B}$ are close to 0 . The magnetic moment of $1.6 \mu_{B}$ for nickel is lower from the expected value by almost a third. However, those results are nearly identical to other theoretical values found in literature, including those obtained by the B3LYP functional [4, 20, 33]. Magnetic moments are strongly localized as is visible on the spin charge density maps shown in Figures 7, 8, 9, 10, 11 and 12.
Tab. 8. Magnetic moments $M_{m}$ in units $\mu_{B}$ for the whole ring, in the interstitial region and for specific magnetic ions obtained within augmented symmetry. For all cases two spin configurations are shown - antiferromagnetic (AF) and ferromagnetic (FM). Site numbering follows that in Fig. 3

\begin{tabular}{l|r|r|r|r|r|r}
\cline { 2 - 7 } \multicolumn{1}{c||}{} & \multicolumn{2}{c|}{$\mathrm{Cr}_{8}$} & \multicolumn{2}{c|}{$\mathrm{Cr}_{7} \mathrm{Cd}$} & \multicolumn{2}{c}{$\mathrm{Cr}_{7} \mathrm{Ni}$} \\
\hline$M_{m}$ & $\mathrm{AF}$ & $\mathrm{FM}$ & $\mathrm{AF}$ & $\mathrm{FM}$ & $\mathrm{AF}$ & $\mathrm{FM}$ \\
\hline \hline total & 0.00 & 24.00 & -3.00 & 21.00 & -1.00 & 23.00 \\
\hline int. & -0.01 & 1.42 & -0.19 & 1.24 & -0.09 & 1.33 \\
\hline $\mathrm{M} 1$ & 2.73 & 2.78 & -0.01 & 0.01 & 1.59 & 1.62 \\
\hline $\mathrm{Cr} 2$ & -2.73 & 2.77 & -2.74 & 2.76 & -2.72 & 2.77 \\
\hline $\mathrm{Cr} 3$ & 2.73 & 2.78 & 2.73 & 2.79 & 2.73 & 2.79 \\
\hline $\mathrm{Cr} 4$ & -2.72 & 2.77 & -2.73 & 2.78 & -2.73 & 2.78 \\
\hline $\mathrm{Cr} 5$ & 2.73 & 2.78 & 2.74 & 2.78 & 2.74 & 2.78 \\
\hline
\end{tabular}

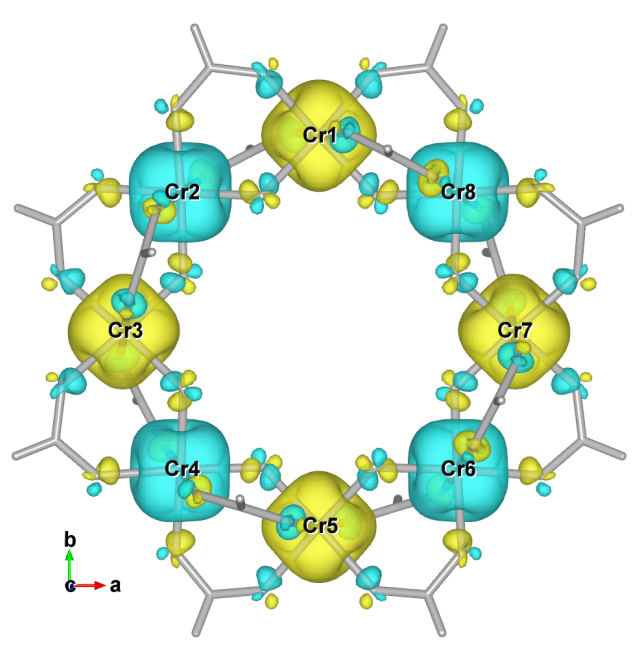

Fig. 7. Plot of the spin density for the AF configuration of $\mathrm{Cr}_{8}$ molecule. Yellow (blue) shades are positive (negative) isosurfaces for the value $0.01 e / r_{\mathrm{Bohr}}^{3}$ 


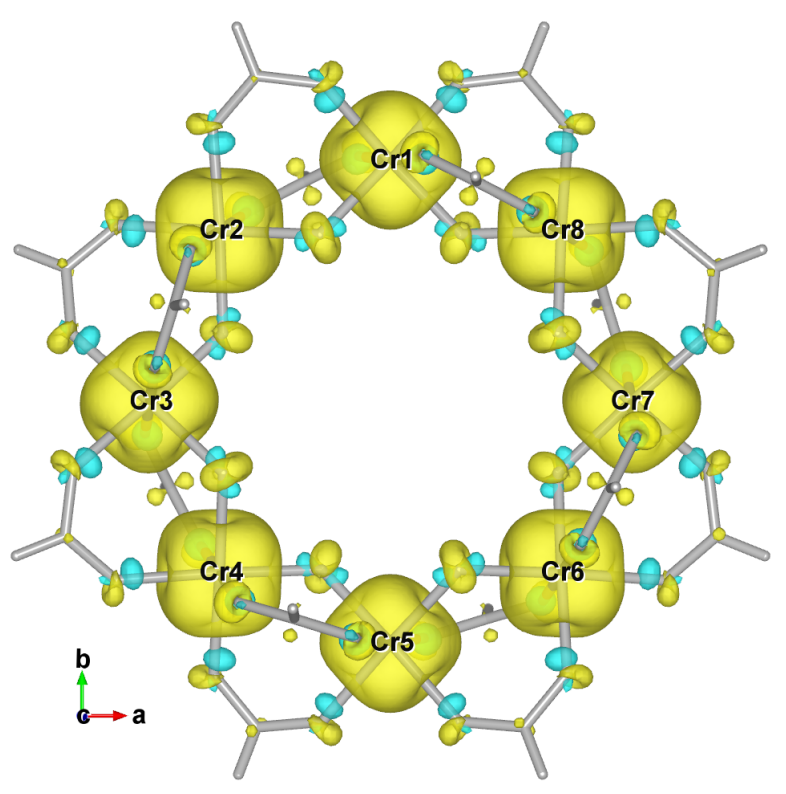

Fig. 8. Plot of the spin density for the FM configuration of $\mathrm{Cr}_{8}$ molecule. Yellow (blue) shades are positive (negative) isosurfaces for the value $0.01 e / r_{\mathrm{Bohr}}^{3}$

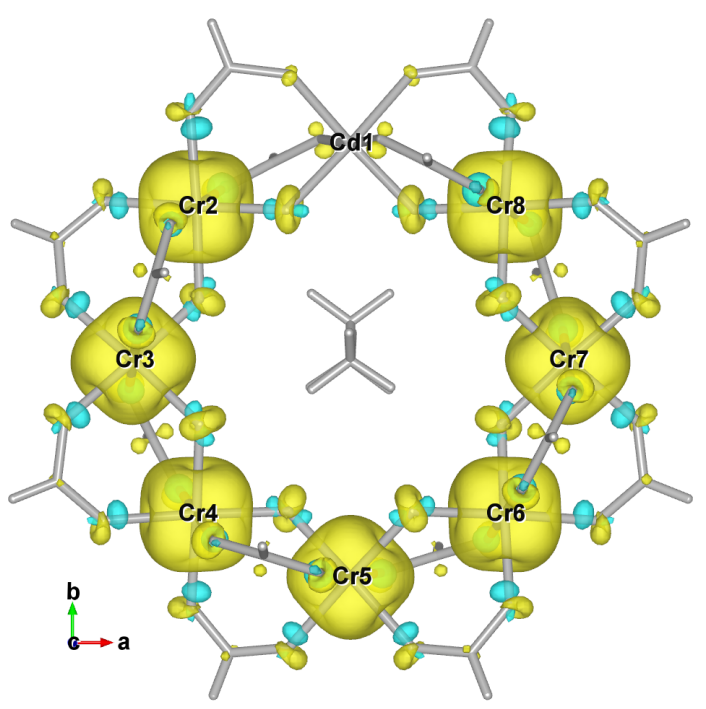

Fig. 10. Plot of the spin density for the FM configuration of $\mathrm{Cr}_{7} \mathrm{Cd}$ molecule. Yellow (blue) shades are positive (negative) isosurfaces for the value $0.01 e / r_{\mathrm{Bohr}}^{3}$

The H-L gaps calculated for minority (min.) and majority (maj.) channels are presented in Tab. 9. For $\mathrm{Cr}_{8}$ they are consistent with those presented in $[9,20,25]$ and vary in a range of $10 \%$. For the heteronuclear derivatives our val-

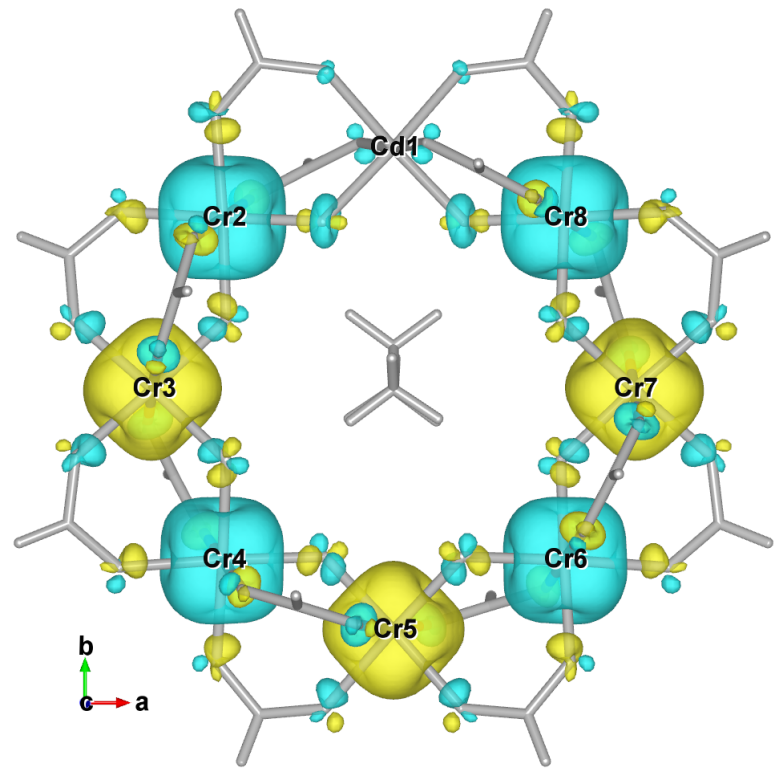

Fig. 9. Plot of the spin density for the AF configuration of $\mathrm{Cr}_{7} \mathrm{Cd}$ molecule. Yellow (blue) shades are positive (negative) isosurfaces for the value $0.01 e / r_{\mathrm{Bohr}}^{3}$

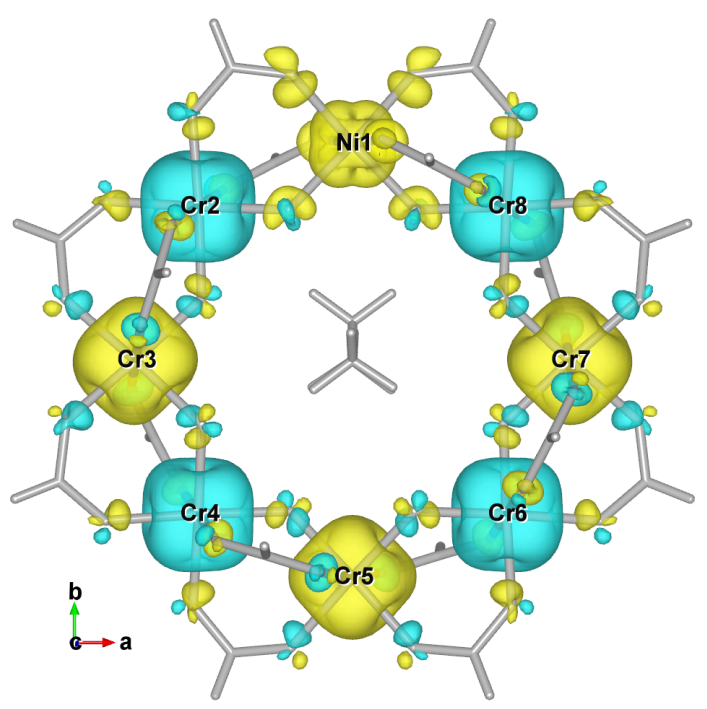

Fig. 11. Plot of the spin density for the AF configuration of $\mathrm{Cr}_{7} \mathrm{Ni}$ molecule. Yellow (blue) shades are positive (negative) isosurfaces for the value $0.01 e / r_{\text {Bohr }}^{3}$

ues are around 6 times larger, but we were unable to find theoretical results for PBE functional apart from the earlier results obtained using the SIESTA package [25] which may not be reliable due to difficulties encountered in choosing the 
proper pseudopotentials for the $\mathrm{Cd}$ and $\mathrm{Ni}$ ions as well as to the approximate charge balancing procedure applied.

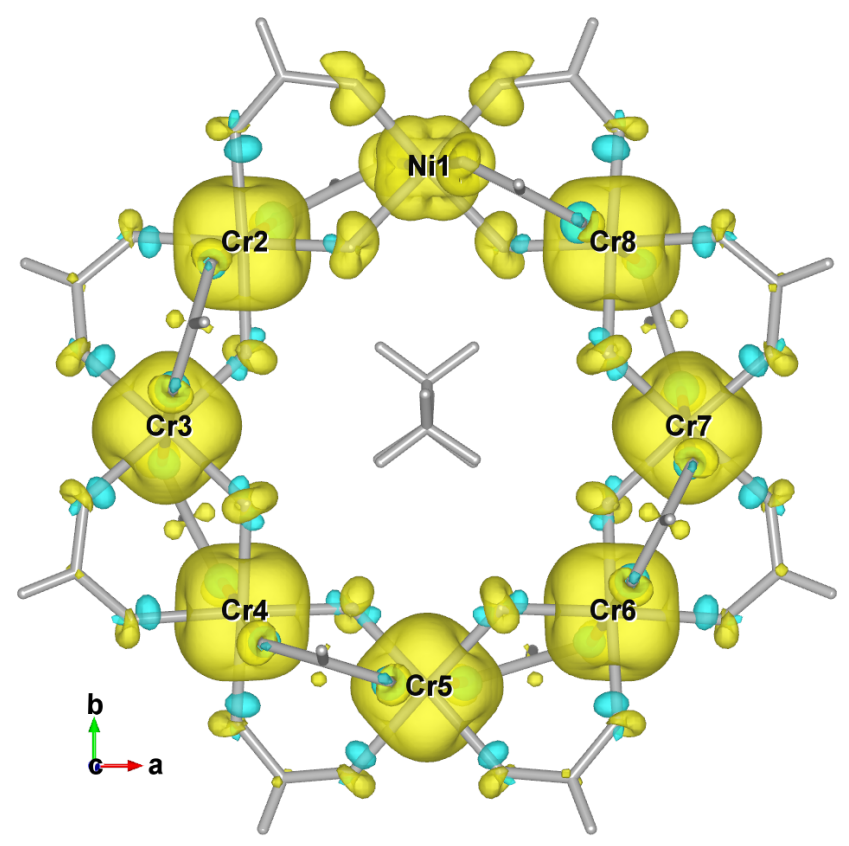

Fig. 12. Plot of the spin density for the FM configuration of $\mathrm{Cr}_{7} \mathrm{Ni}$ molecule. Yellow (blue) shades are positive (negative) isosurfaces for the value $0.01 e / r_{\mathrm{Bohr}}^{3}$

Tab. 9. HOMO-LUMO majority and minority gaps for the $\mathrm{Cr}_{7} \mathrm{M}$ molecules calculated for the AF and FM configurations in the AS approach. For reference other literature values are given: FS-based obtained by WIEN2k $[9,20]$ and NS-based found by using the SIESTA package [25]

\begin{tabular}{|c|c|c|c|c|}
\hline \multicolumn{2}{|c|}{ H-L gap } & $\mathrm{Cr}_{8}$ & $\mathrm{Cr}_{7} \mathrm{Cd}$ & $\mathrm{Cr}_{7} \mathrm{Ni}$ \\
\hline \multirow{2}{*}{$\mathrm{AF}(\mathrm{AS})$} & $E_{\min }$. & 2.38 & 0.97 & 0.75 \\
\hline & $E_{\text {maj. }}$ & 2.42 & 0.99 & 0.88 \\
\hline \multirow{2}{*}{ FM (AS) } & $E_{\min }$. & 3.77 & 1.01 & 0.85 \\
\hline & $E_{\text {maj. }}$ & 2.18 & 0.80 & 0.74 \\
\hline \multirow{2}{*}{$\mathrm{AF}[20]$} & $E_{\min }$. & 2.35 & & \\
\hline & $E_{\text {maj. }}$. & 2.35 & & \\
\hline \multirow{2}{*}{ FM [20] } & $E_{\min }$ & 3.88 & & \\
\hline & $E_{\text {maj. }}$ & 2.31 & & \\
\hline \multirow{2}{*}{$\mathrm{AF}[9]$} & $E_{\min }$. & 2.36 & & \\
\hline & $E_{\text {maj. }}$ & 2.36 & & \\
\hline \multirow{2}{*}{ FM [9] } & $E_{\min }$ & 4.08 & & \\
\hline & $E_{\text {maj. }}$ & 2.15 & & \\
\hline $\mathrm{AF}$ [25] & $E_{\text {maj. }}$ & 2.38 & 0.15 & 0.18 \\
\hline FM [25] & $E_{\text {maj. }}$ & 2.01 & 0.11 & 0.13 \\
\hline
\end{tabular}

\section{2. Exchange couplings}

The coupling parameters obtained for the basis set $\mathrm{RKM}=3.0$ are shown in Tab. 10. They compare very well with other theoretical predictions found in literature and based on the PBE functional $[20,33]$. Any difference between our $J$ values is well within standard variation encountered among other DFT estimates [4, 8, 25, 29, 34-38]. The discrepancies can be neglected, because all the DFT values are significantly overestimated. Not only are the DFT values of $J$ overestimated, but even the relations between $J$ and $J^{\prime}$ are not correctly reproduced. This overestimation problem is persistent and clearly cannot be overcome with simple symmetry modification provided by the AS approach. We emphasize that extraction of the magnetic couplings is a challenge and within PBE they are known to deviate significantly from experimental findings. For the purpose of this paper it is important that the $J$ estimates found here are comparable to other PBE-based data.

Tab. 10. Exchange couplings obtained for $\mathrm{Cr}_{7} \mathrm{M}$ molecules within the AS approach. All the values in units of meV. For reference other theoretical values are given: the data of the FS approach in WIEN2k [20] and NS approach in SIESTA [33]. Experimental values estimated from inelastic neutron scattering (INS) and electron paramagnetic resonance (EPR) are also shown [32, 39-41]

\begin{tabular}{l||r|r|r|r|r}
\cline { 2 - 6 } \multicolumn{1}{c||}{} & $\mathrm{Cr}_{8}$ & $\mathrm{Cr}_{7} \mathrm{Cd}$ & \multicolumn{3}{|c}{$\mathrm{Cr}_{7} \mathrm{Ni}$} \\
\hline source & $J$ & $J$ & $J$ & $J^{\prime}$ & $J^{\prime} / J$ \\
\hline \hline DFT, this paper & 6.7 & 7.0 & 7.0 & 2.3 & 0.33 \\
\hline DFT [20] & 6.3 & & & & \\
\hline DFT [33] & & 6.9 & 6.9 & 5.1 & 0.74 \\
\hline INS [39, 40] & 1.46 & & & & \\
\hline INS [41] & & 1.46 & 1.46 & 1.69 & 1.16 \\
\hline EPR [32] & & & 1.42 & 2.14 & 1.51 \\
\hline
\end{tabular}

The AS approach constitutes an analogue of a linear model suggested for the chromium rings in $[2,37]$. In the linear model some segments of the rings are cut out and joined to form a linear chain. In the framework of AS idea, a half of a ring is extracted and rotated by $180^{\circ}$ around the axis lying in the plane of the molecule. In this way the final size and shape of the molecule considered is preserved.

An important advantage of the AS approach with respect to FS is the higher number of independent spin configurations which can be analyzed. The enhanced number of configurations is a prerequisite to calculate the magnetic couplings in heterometallic $\mathrm{Cr}_{7} \mathrm{M}$ or non-uniform homometallic $\mathrm{Cr}_{9}$ rings [23, 38].

The energies of all nonequivalent spin configurations related to the $\mathrm{AF}$ ground state found in the $\mathrm{AS}$ approach are presented in Tables 11, 12 and 13 for $\mathrm{Cr}_{8}, \mathrm{Cr}_{7} \mathrm{Ni}$ and $\mathrm{Cr}_{7} \mathrm{Cd}$, respectively. In the second column the string of arrows determines the spin configuration of the ions, where the arrows are aligned according to the enumeration in Fig. 3. 
Tab. 11. Energies of $\mathrm{Cr}_{8}$ ring for all nonequivalent spin configurations of ions taken with respect to the ground state energy corresponding to the AF alignment

\begin{tabular}{lc||c}
\hline $\begin{array}{c}\text { configuration } \\
\text { designation }\end{array}$ & $\begin{array}{c}\text { spin } \\
\text { alignment }\end{array}$ & $\begin{array}{c}E \\
{[\mathrm{meV}]}\end{array}$ \\
\hline \hline$r 0$ (FM) & $\uparrow \uparrow \uparrow \uparrow \uparrow$ & 242.0 \\
$r 12$ & $\downarrow \downarrow \uparrow \uparrow \uparrow$ & 186.3 \\
$r 1$ & $\downarrow \uparrow \uparrow \uparrow \uparrow$ & 182.3 \\
$r 2$ & $\uparrow \downarrow \uparrow \uparrow \uparrow$ & 125.7 \\
$r 3$ & $\uparrow \uparrow \downarrow \uparrow \uparrow$ & 122.9 \\
$r 23$ & $\uparrow \downarrow \downarrow \uparrow \uparrow$ & 119.8 \\
$r 13$ & $\downarrow \uparrow \downarrow \uparrow \uparrow$ & 62.5 \\
$r 14$ & $\downarrow \uparrow \uparrow \downarrow \uparrow$ & 59.3 \\
$r 24$ (AF) & $\uparrow \downarrow \uparrow \downarrow \uparrow$ & 0.0 \\
\hline \multicolumn{3}{c}{} \\
\hline
\end{tabular}

Tab. 12. Energies of $\mathrm{Cr}_{7} \mathrm{Ni}$ ring for all nonequivalent spin configurations of ions taken with respect to the ground state energy corresponding to the $\mathrm{AF}$ alignment

\begin{tabular}{lc||r}
\hline $\begin{array}{c}\text { configuration } \\
\text { designation }\end{array}$ & $\begin{array}{c}\text { spin } \\
\text { alignment }\end{array}$ & $\begin{array}{c}E \\
\text { [meV] }\end{array}$ \\
\hline \hline$n 0$ (FM) & $\uparrow \uparrow \uparrow \uparrow \uparrow$ & 204.4 \\
$n 1$ & $\downarrow \uparrow \uparrow \uparrow \uparrow$ & 189.5 \\
$n 5$ & $\uparrow \uparrow \uparrow \uparrow \downarrow$ & 145.6 \\
$n 45$ & $\uparrow \uparrow \uparrow \downarrow \downarrow$ & 138.8 \\
$n 12$ & $\downarrow \downarrow \uparrow \uparrow \uparrow$ & 138.0 \\
$n 15$ & $\downarrow \uparrow \uparrow \uparrow \downarrow$ & 130.5 \\
$n 2$ & $\uparrow \downarrow \uparrow \uparrow \uparrow$ & 125.8 \\
$n 23$ & $\uparrow \downarrow \downarrow \uparrow \uparrow$ & 122.9 \\
$n 4$ & $\uparrow \uparrow \uparrow \downarrow \uparrow$ & 79.9 \\
$n 34$ & $\uparrow \uparrow \downarrow \downarrow \uparrow$ & 80.2 \\
$n 3$ & $\uparrow \uparrow \downarrow \uparrow \uparrow$ & 71.5 \\
$n 25$ & $\uparrow \downarrow \uparrow \uparrow \downarrow$ & 67.8 \\
$n 14$ & $\downarrow \uparrow \uparrow \downarrow \uparrow$ & 63.9 \\
$n 13$ & $\downarrow \uparrow \downarrow \uparrow \uparrow$ & 58.9 \\
$n 35$ (AM) & $\uparrow \uparrow \downarrow \uparrow \downarrow$ & 12.6 \\
$n 24$ (AF) & $\uparrow \downarrow \uparrow \downarrow \uparrow$ & 0.0 \\
\hline
\end{tabular}

Tab. 13. Energies of $\mathrm{Cr}_{7} \mathrm{Cd}$ ring for all nonequivalent spin configurations of ions taken with respect to the ground state energy corresponding to the AF alignment. The first arrow in the second column is missing due to vanishing spin on the $\mathrm{Cd}$ center

\begin{tabular}{lc||r}
\hline $\begin{array}{c}\text { configuration } \\
\text { designation }\end{array}$ & $\begin{array}{c}\text { spin } \\
\text { alignment }\end{array}$ & $\begin{array}{c}E \\
\text { [meV] }\end{array}$ \\
\hline \hline$d 0$ (FM) & $-\uparrow \uparrow \uparrow \uparrow$ & 189.4 \\
$d 5$ & $-\uparrow \uparrow \uparrow \downarrow$ & 130.6 \\
$d 2$ & $-\downarrow \uparrow \uparrow \uparrow$ & 125.5 \\
$d 23$ & $-\downarrow \downarrow \uparrow \uparrow$ & 123.9 \\
$d 34$ & $-\uparrow \downarrow \downarrow \uparrow$ & 66.7 \\
$d 4$ & $-\uparrow \uparrow \downarrow \uparrow$ & 65.0 \\
$d 3$ & $-\uparrow \downarrow \uparrow \uparrow$ & 58.9 \\
$d 24$ (AF) & $-\downarrow \uparrow \downarrow \uparrow$ & 0.0 \\
\hline
\end{tabular}

\section{CONCLUSIONS}

We have carried out the first principle systematic DFT calculations for the $\mathrm{Cr}_{7} \mathrm{M}$ family of molecular nanorings using WIEN2k computational package and augmented symmetry approach by having recourse to the PBE functional. We have extracted the electronic and magnetic properties of these molecules. The obtained coupling parameters $J$, HOMO-LUMO gaps and magnetic moments compare very well with other DFT theoretical predictions found in literature.

We have shown that the AS model leads to the results that are consistent with other PBE-based approaches, are numerically stable and reliable for $\mathrm{RKM}=3.0$ and a single k-point. The features of AS were tested by variation of RKM, k-mesh size and unit cell. However, the most important fact is that the augmented symmetry approach is computationally much less demanding than the NS counterpart and allows for significant improvement of computational performance, almost identical to that of the FS treatment. Accordingly, AS enables conducting calculations that otherwise would be non-feasible due to the excessively high computational complexity.

The AS model discussed in this paper was originally devised to mimic the even-numbered molecules. However, after slight modifications it can be applied to a family of odd-numbered chromium ring molecules $\mathrm{Cr}_{8} \mathrm{M}$ [36]. We also intend to use the AS approach in conjunction with hybrid Becke 3-term Lee-Yang-Parr (B3LYP) correlation functional $[42,43]$ which is known to improve the $J$ parameter estimates $[4,20]$.

\section{Acknowledgments}

One of the authors (M.W.) was supported by the European Union scholarship No. DFS.VI.052.4.62.8.2013 from Human Capital Programme, sub-measure 8.2.2, priority VIII, funded from EFS and the Lubusz Voivodeship budget. Calculations were performed on the HPC resources in PSNC Poznań (Poland). Preliminary tests were carried on computing servers in the Institute of Physics at University of Zielona Góra.

\section{References}

[1] M. Wojciechowski, B. Brzostowski, G. Kamieniarz, Augmented Symmetry Approach to the DFT Simulations of the Chromium-Based Rings, in: R. Wyrzykowski et al. (ed.) Parallel Processing and Applied Mathematics, 11th International Conference PPAM 2015, Berlin Heidelberg Springer-Verlag, p. 321-331, 2016.

[2] D. M. Tomecka, V. Bellini, F. Troiani, F. Manghi, G. Kamieniarz, M. Affronte, Ab initio study of a chain model of the Cr $r_{8}$ molecular magnet, Phys. Rev. B 77224401 (2008). 
[3] V. Corradini, F. Moro, R. Biagi, V. De Renzi, U. del Pennino, V. Bellini, S. Carretta, P. Santini, V. A. Milway, G. Timco, R. E. P. Winpenny, M. Affronte, Successful grafting of isolated molecular $\mathrm{Cr}_{7} \mathrm{Ni}$ rings on Au(111) surface, Phys. Rev. B 79, 144419 (2009).

[4] V. Bellini, M. Affronte, A density-functional study of heterometallic Cr-based molecular rings, J. Phys. Chem. B 114, 14797 (2010).

[5] J. van Slageren, R. Sessoli, D. Gatteschi, A. A. Smith, M. Helliwell, R. E. P. Winpenny, A. Cornia, A.-L. Barra, A. G. M. Jansen, E. Rentschler, G. Timco, Magnetic anisotropy of the antiferromagnetic ring $\mathrm{Cr}_{8} \mathrm{~F}_{8} \mathrm{Piv}_{16}$, Chem. Eur. J. 8, 277 (2002).

[6] F. K. Larsen, E. J. L. McInnes, H. E. Mkami, J. Overgaard, S. Piligkos, G. Rajaraman, E. Rentschler, A. A. Smith, G. M. Smith, V. Boote, M. Jennings, G. A. Timco, R. E. P. Winpenny, Synthesis and characterization of heterometallic $\mathrm{Cr}_{7} M$ wheels, Angew. Chem. Int. Ed. 42, 101 (2003).

[7] C. R. Groom, I. J. Bruno, M. P. Lightfoot, S. C. Ward, The Cambridge Structural Database, Acta Cryst. B72, 171 (2016).

[8] T. Ślusarski, B. Brzostowski, D. M. Tomecka, G. Kamieniarz, Application of the package SIESTA to linear models of a molecular chromium-based ring, Acta Phys. Pol. A. 118, 967 (2010).

[9] V. Bellini, A. Olivieri, F. Manghi, Density-functional study of the $\mathrm{Cr}_{8}$ antiferromagnetic ring, Phys. Rev. B 73, 184431 (2006).

[10] P. Hohenberg, W. Kohn, Inhomogeneous Electron Gas, Phys. Rev. 136, 864 (1964).

[11] W. Kohn, L. J. Sham, Self-Consistent Equations Including Exchange and Correlation Effects, Phys. Rev. 140, A1133 (1965).

[12] P. Blaha, K. Schwarz, G. H. K. Madsen, D. Kvasnicka, J. Luitz, WIEN2k: An Augmented Plane Wave + Local Orbitals Program for Calculating Crystal Properties, Karlheinz Schwarz, Techn. Universität, Wien (1999).

[13] E. Sjöstedt, L. Nordström, D. J. Singh, An alternative way of linearizing the augmented plane-wave method, Solid State Commun. 114, 15 (2000).

[14] G. K. H. Madsen, P. Blaha, K. Schwarz, E. Sjöstedt, L. Nordström, Efficient linearization of the augmented plane-wave method, Phys. Rev. B 64, 195134 (2001).

[15] O. K. Andersen, Linear methods in band theory, Phys. Rev. B 12, 3060 (1975).

[16] D. J. Singh, Planewaves Pseudopotentials and the LAPW Method, Kluwer Academic Publishers, Boston (1994).

[17] J. P. Desclaux, Hartree Fock Slater self consistent field calculations, Comp. Phys. Commun. 1, 216 (1969).

[18] D. D. Koelling, B. N. Harmon, A technique for relativistic spin-polarised calculations, J. Phys. C: Sol. St. Phys. 10, 3107 (1977).

[19] J. C. Slater, Wave Functions in a Periodic Potential, Phys. Rev. 51, 846 (1937).

[20] M. Wojciechowski, B. Brzostowski, G. Kamieniarz, DFT estimation of exchange coupling constant of $\mathrm{Cr}_{8}$ molecular ring using the hybrid functional B3LYP, Acta Phys. Pol. A. 127, 407 (2015).

[21] J. P. Perdew, K. Burke, M. Ernzerhof, Generalized gradient approximation made simple, Phys. Rev. Lett. 77, 3865 (1996).

[22] M. L. Baker, T. Guidi, S. Carretta, J. Ollivier, H. Mutka, H. U. Güdel, G. A. Timco, E. J. L. McInnes, G. Amoretti, R. E. P. Winpenny, P. Santini, Spin dynamics of molecular nanomagnets unravelled at atomic scale by four-dimensional inelastic neutron scattering, Nat. Phys. 8, 906 (2012).
[23] M. Antkowiak, P. Kozłowski, G. Kamieniarz, G. A. Timco, F. Tuna, R. E. P. Winpenny, Detection of ground states in frustrated molecular rings by in-field local magnetization profiles, Phys. Rev. B 87, 184430 (2013).

[24] L. Kronik, T. Stein, S. Refaely-Abramson, R. Baer, Excitation Gaps of Finite-Sized Systems from Optimally Tuned RangeSeparated Hybrid Functionals, J. Chem. Theory Comput. 8, 1515 (2012).

[25] B. Brzostowski, M. Wojciechowski, G. Kamieniarz, Fundamental gaps in $\mathrm{Cr}_{8}, \mathrm{Cr}_{7} \mathrm{Ni}$ and $\mathrm{Cr}_{7} \mathrm{Cd}$ molecules, Acta Phys. Pol. A. 126, 234 (2014).

[26] H. Mera, K. Stokbro, Using Kohn-Sham density functional theory to describe charged excitations in finite systems, Phys. Rev. B 79, 125109 (2009).

[27] X. Andrade, A. Aspuru-Guzik, Prediction of the Derivative Discontinuity in Density Functional Theory from an Electrostatic Description of the Exchange and Correlation Potential, Phys. Rev. Lett. 107, 183002 (2011).

[28] L. J. Noodleman, Valence bond description of antiferromagnetic coupling in transition metal dimers, Chem. Phys. 74, 5737 (1981).

[29] G. Kamieniarz, P. Kozłowski, M. Antkowiak, P. Sobczak, T. Ślusarski, D. M. Tomecka, A. Barasiński, B. Brzostowski, A. Drzewiński, A. Bieńko, J. Mroziński, Anisotropy, geometric structure and frustration effects in molecule-based nanomagnets, Acta Phys. Pol. A. 121, 992 (2012).

[30] P. Blaha, K. Schwarz, G. H. K. Madsen, D. Kvasnicka, J. Luitz, WIEN2k User's Guide; revision 14.2 (Release 10/15/2014), www.wien2k.at/reg_user/textbooks/.

[31] P. Blaha, K. Schwarz, J. Luitz, WIEN2k: Frequently asked Questions,www.wien2k.at/reg_user/faq/.

[32] S. Piligkos, H. Weihe, E. Bill, F. Neese, H. E. Mkami, G. M. Smith, D. Collison, G. Rajaraman, G. A. Timco, R. E. P. Winpenny, E. J. L. McInnes, EPR Spectroscopy of a Family of $\mathrm{Cr}^{I I}{ }_{7} \mathrm{M}^{I I}(\mathrm{M}=\mathrm{Cd}, \mathrm{Zn}, \mathrm{Mn}$, Ni) Wheels: Studies of Isostructural Compounds with Different Spin Ground States, Chem. Eur. J. 15, 3152 (2009).

[33] B. Brzostowski, R. Lemański, T. Ślusarski, D. Tomecka, G. Kamieniarz, Chromium-based rings within the DFT and Falicov-Kimball model approach, J. Nanopart. Res. 15, 1528 (2013).

[34] B. Brzostowski, T. Ślusarski, G. Kamieniarz, DFT study of octanuclear molecular chromium-based ring using new pseudopotential parameters, Acta Phys. Pol. A. 121, 1115 (2012).

[35] B. Brzostowski, M. Wojciechowski, R. Lemański, G. Kamieniarz, DFT and Falicov-Kimball model approach to Cr9 molecular ring, Acta Phys. Pol. A. 126, 270 (2014).

[36] M. Wojciechowski, B. Brzostowski, R. Lemański, G. Kamieniarz, Mapping of the DFT spin configuration energies of $\mathrm{Cr} 8 \mathrm{Cd}$ molecular ring onto the energy structure of Falicov-Kimball model, Acta Phys. Pol. A. 127, 410 (2015).

[37] V. Bellini, D.M. Tomecka, B. Brzostowski, M. Wojciechowski, F. Troiani, F. Manghi, M. Affronte, DFT study of the $\mathrm{Cr}_{8}$ molecular magnet within chain-model approximations, in: R. Wyrzykowski et al. (ed.) Parallel Processing and Applied Mathematics, 10th International Conference PPAM 2013, Berlin Heidelberg Springer-Verlag, p. 428-437, 2014.

[38] M. L. Baker, G. A. Timco, S. Piligkos, J. S. Mathieson, H. Mutka, F. Tuna, P. Kozłowski, M. Antkowiak, T. Guidi, T. Gupta, H. Rath, R. J. Woolfson, G. Kamieniarz, R. G. Pritchard, H. Weihe, L. Cronin, G. Rajaraman, D. Collison, E. J. L. McInnes, R. E. P. Winpenny, A classification of spin frustration in molecular magnets from a physical study of large odd-numbered-metal, odd electron rings, Proc. Natl. Acad. Sci. U.S.A. 109, 19113 (2012). 
[39] P. Christian, G. Rajaraman, A. Harrison, J. J. W. McDouall, J. T. Rafterya, R. E. P. Winpenny, Structural, magnetic and DFT studies of a hydroxide-bridged $\mathrm{Cr}_{8}$ wheel, Dalton Trans. 10, 1511 (2004).

[40] S. Carretta, J. van Slageren, T. Guidi, E. Liviotti, C. Mondelli, D. Rovai, A. Cornia, A. L. Dearden, F. Carsughi, M. Affronte, C. D. Frost, R. E. P. Winpenny, D. Gatteschi, G. Amoretti, R. Caciuffo, Microscopic spin Hamiltonian of a $\mathrm{Cr}_{8}$ antiferromagnetic ring from inelastic neutron scattering, Phys. Rev. 67, 094405 (2003).
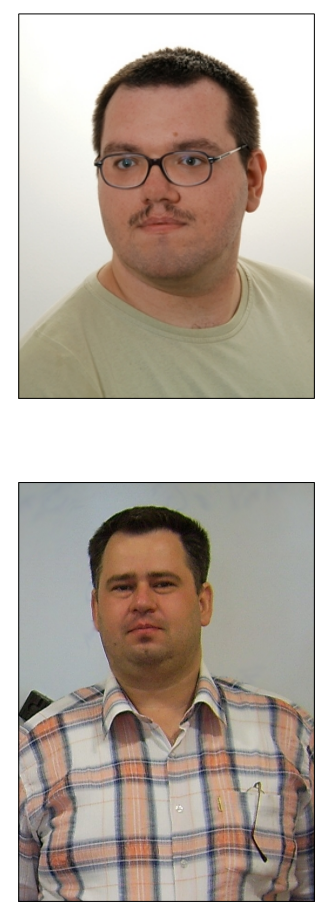

Michał Wojciechowski holds master degree in Physics obtained at Faculty of Physics and Astronomy, University of Zielona Góra. At present he is $\mathrm{PhD}$ student at this faculty and prepares his thesis under the supervision of Grzegorz Kamieniarz.

Bartosz Brzostowski works at the Institute of Physics of the University of Zielona Góra, Poland. He received $\mathrm{PhD}$ degree in Physics in 2003 from University of Wrocław. His main areas of interest include quantum optics, solid state physics and several topics in molecular physics (mainly magnetic molecules).

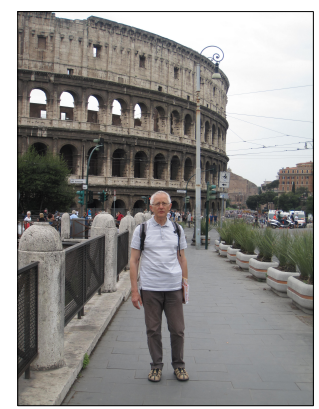

Grzegorz Kamieniarz is a professor in theoretical and computational physics at the Institute of Physics of the Adam Mickiewicz University in Poznań. His field of interest covers statistical mechanics, phase transitions, phenomenological modeling and simulations of the low-dimensional magnetic systems including molecular nanomagnets. 